\title{
THE COMPARE OF OXYGEN UPTAKE KINETICS OF YOUNG SOCCER PLAYERS ACCORDING TO PLAY POSITIONS
}

\author{
${ }^{1}$ Hamit CIHAN, ${ }^{2}$ Erdal ARI, ${ }^{3}$ Ibrahim CAN, ${ }^{4}$ Bahadır DEMIR \\ ${ }^{1}$ Karadeniz Technical University Physical Education and Sports School, Turkey. \\ ${ }^{2}$ Ordu University Physical Education and Sports School, Turkey. \\ ${ }^{3}$ Gümüşhane University Physical Education and Sports School, Turkey. \\ ${ }^{4}$ Physical Education Teacher, Ministry of the National Education, Turkey.
}

\begin{abstract}
The purpose of this study was to compare oxygen uptake kinetics, ventilatory threshold of young soccer players according to playing position and to determine relationship between oxygen uptake kinetics and ventilatory threshold of young soccer players. Twenty-three young soccer players joined to study voluntarily ( $\mathrm{n}=23$; age: $19.8 \pm 0.4$ years, body height: $179.4 \pm 7.2 \mathrm{~cm}$., body mass: $74.2 \pm 7.4 \mathrm{~kg}$., $\mathrm{VO}_{2} \max : 59.7 \pm 8 \mathrm{ml} / \mathrm{kg} / \mathrm{min}$.). The players were categorized according to playing positions. The $\mathrm{VO}_{2} \max$, ventilatory threshold were determined by incremental treadmill test. Then, treadmill test at running velocity determined $\mathrm{VO}_{2}$ max was performed and oxygen uptake kinetics were identified by mono-exponentinal model. The time to achieve $95 \%$ of $\mathrm{VO}_{2}$ max, spending time at $\mathrm{VO}_{2} \mathrm{max}$ were accepted as oxygen uptake kinetics. The correlation among time to achieve $95 \%$ of $\mathrm{VO}_{2} \mathrm{max}$, spending time at $\mathrm{VO}_{2} \max$ and ventilatory threshold values of young soccer were determined by correlation anlyze and differences according to playing positions were identified by one-way analysis of variance. It was determined significiant negative correlation between time to achieve $95 \%$ of $\mathrm{VO}_{2} \max$ and spending time at $\mathrm{VO}_{2} \max (\mathrm{r}=-0.526$, $\mathrm{p}<0.05$ ). The no significiant difference among playing positions was found without time to achieve $95 \%$ of $\mathrm{VO}_{2}$ max. The centerbacks had higher time to achieve $95 \%$ of $\mathrm{VO}_{2}$ max values than goalkeepers $(\mathrm{p}<0.05)$ but no significiant difference among other playing positions was found ( $>0.05)$. Consequently, it could be said that reaching to steady-state level early during exercise could shorten time to achieve $95 \%$ of $\mathrm{VO}_{2}$ max and increase spending time at $\mathrm{VO}_{2} \max$.
\end{abstract}

Key Words: Oxygen uptake kinetics, soccer, play position.

\section{GENÇ FUTBOL OYUNCULARININ OKSİJEN TÜKETIMI KINETIKLERININ MEVKILERINE GÖRE KARŞILAŞTIRILMASI}

\begin{abstract}
Özet
$\mathrm{Bu}$ çalışmanın amacı genç futbol oyuncularının oksijen tüketimi kinetikleri ile solunum eşiği değerleri arasındaki ilişkiyi belirlemek ve oyuncuların mevkilerine göre oksijen tüketimi kinetikleri ve solunum eşiği değerlerini karşılaş̧ırmaktır. 23 genç futbol oyuncusu gönüllü olarak çalışmaya katılmıştır $(\mathrm{n}=23$, yaş: $19.8 \pm 0.4$ yıl, boy uzunluğu: $179.4 \pm 7.2 \mathrm{~cm}$., vücut ağıllığ: $74.2 \pm 7.4 \mathrm{~kg}$., $\mathrm{VO}_{2} \max : 59.7 \pm 8 \mathrm{ml} / \mathrm{kg} / \mathrm{dk}$.). Maksimum oksijen tüketimi $\left(\mathrm{VO}_{2} \max \right)$ ve solunum eşiği değerleri, koşu bandında uygulanan ve koşu hızı giderek artan test protokolüyle belirlenmiştir. Daha sonra $\mathrm{VO}_{2}$ max değerine tekabül eden koşu hızında test uygulanmıştır ve oksijen tüketim kinetiği değerleri mono-exponentinal model yardımıyla belirlenmiştir. $\mathrm{VO}_{2}$ max değerinin $\% 95$ 'ine ulaşılan süre ve $\mathrm{VO}_{2} \max$ değerinde geçirilen süre, oksijen tüketimi kinetikleri olarak kabul edilmiştir. $\mathrm{VO}_{2}$ max değerinin $\%$ 95'ine ulaşılan süre, $\mathrm{VO}_{2}$ max değerinde geçirilen süre ve solunum eşiği değerleri arasındaki ilişkiler korelasyon analiziyle, mevkilere göre farklılıklar ise tek yönlü varyans analiziyle belirlenmiştir. $\mathrm{VO}_{2}$ max değerinin \% 95'ine ulaşılan süre ile $\mathrm{VO}_{2}$ max değerinde geçirilen süre arasında negatif yönlü ve anlamlı bir ilişki tespit edilmiştir ( $\mathrm{r}=-$ 0.526, $\mathrm{p}<0.05) . \mathrm{VO}_{2} \max$ değerinin $\% 95$ 'ine ulaşılan süre hariç olmak üzere diğer değerler bakımından oyuncuların mevkileri arasında anlamlı farklılık görülmemiştir. $\mathrm{VO}_{2} \max$ değerinin \% 95'ine ulaşılan süre bakımından stoper oyuncularının kalecilerden daha yüksek değerlere sahip olduğu $(\mathrm{p}<0.05)$, diğer mevkiler arasında ise herhangi bir farklılık olmadığı belirlenmiştir. Sonuç olarak egzersizde steady-state seviyesine kısa zamanda ulaşmanın $\mathrm{VO}_{2}$ max değerinin \% 95'ine ulaşılan süreyi kısaltabileceği ve $\mathrm{VO}_{2}$ max değerinde geçirilen süreyi arttırabileceği ifade edilebilir.
\end{abstract}

Anahtar Kelimeler: Oksijen tüketim kinetikleri, futbol, mevki. 


\section{Introduction}

The capacity of oxygen using during exercise was important for performance. Maximum oxygen uptake $\left(\mathrm{VO}_{2} \max \right)$ was the highest oxygen amount utilized by body during exercise (Bassett and Howley, 2000). $\mathrm{VO}_{2} \max$ is a important parameter effecting aerobic performance of athletes. At initial of incremental exercise, oxygen uptake $\left(\mathrm{VO}_{2}\right)$ increases linearly until steady-state level is achieved. $\mathrm{VO}_{2}$ rises mono-exponentially to reach steady-state level within 2-3 minutes after onset of constant moderate exercise (Carter et al., 2000). The oxygen deficit exists at part between initial of incremental exercise and steady-state level. The amount of oxygen deficit effects reach time to steady state level. Time constant parameter $(\tau)$ is estimated by exponential function during period of reach to steady-state level (Burnley and Jones, 2007) The $\tau$ parameter is equal to $63 \%$ of final $\mathrm{VO}_{2}$ response determined by monoexponential function (Jones and Poole, 2005) The $\tau$ parameter determines amount of oxygen deficit. It means that a smaller value of $\tau$ parameter diminishes reach time to steady state level and is required to anaerobic energy systems. The fatigue will be delayed since it is smally required to support of anaerobic energy systems (Burnley and Jones, 2007).

Soccer is a intermittent sport branch required high level of aerobic fitness parameters. The avarage $\mathrm{VO}_{2}$ max values of elite soccer players was determined between 56.8 and $67.6 \mathrm{ml} / \mathrm{kg} / \mathrm{min}(\mathrm{Al}$ Hazzaa et al., 2001; Árnason et al., 2004; Bangsbo and Lindquist, 1992; Bangsbo et al., 1991; Casajús, 2001; Davis et al., 1992; Rhodes et al., 1986; Strudwick et al., 2002; Wisloeff et al., 1998). Also, it was determined that increase of $\mathrm{VO}_{2} \max$ and running economy $\left(5 \mathrm{ml} / \mathrm{kg}^{\prime} \min\right.$ and $7 \%$, respectively) improved match performance of soccer players (Chamari et al., 2005; Helgerud et al., 2001). The energy costs of activities performed at soccer game such as dribbling with ball are similar to energy costs of laboratory treadmill tests performed with inclination for determining $\mathrm{VO}_{2} \max$ (Kemi et al., 2003). The soccer players having high values of aerobic capacity performs soccer activities with less energy cost and delays fatigue at exercise as $\mathrm{VO}_{2}$ of muscles is high.

Although many studies were performed regarding activity profiles and covered distance values during soccer game of young soccer players, no study was performed regarding $\mathrm{VO}_{2}$ kinetics of young soccer players according to playing position. The investigating of $\mathrm{VO}_{2}$ kinetics of young soccer players according to play position will be useful for developing performances of players, planning of trainings and determining of physiological requirements of play positions. Therefore, aim of this study was to compare $\mathrm{VO}_{2}$ kinetics and ventilatory threshold $(\mathrm{Vt})$ values of young soccer players according to playing position, to determine relationship between $\mathrm{VO}_{2}$ kinetics and $\mathrm{Vt}$ values of young soccer players and to test the hypothesis that $\mathrm{VO}_{2}$ kinetics and $\mathrm{Vt}$ values of young soccer players will diferentiate according to playing positions. 


\section{Method}

\section{Research Group}

Twenty-three young soccer players playing young team category of professional soccer team placing Turkish Super League participated to this study voluntarily ( $\mathrm{n}=23$; age: $19.8 \pm 0.4$ years; body height: $179.4 \pm 7.2 \mathrm{~cm}$; body mass: $74.2 \pm 7.4 \mathrm{~kg}$; $\left.\mathrm{VO}_{2} \max : 59.7 \pm 8 \mathrm{ml} / \mathrm{kg} / \mathrm{min}\right)$. Twenty-three young soccer players were divided to six playing position categories as goalkeepers ( $\mathrm{n}=3$; age: $19.7 \pm 0.5$ years; body height: $186.3 \pm 1.5 \mathrm{~cm}$; body mass: $83.1 \pm 2.8 \mathrm{~kg}$; $\left.\mathrm{VO}_{2} \max : 52.3 \pm 1.2 \mathrm{ml} / \mathrm{kg} / \min \right)$, fullbacks $(\mathrm{n}=4$; age: $19.8 \pm 0.5$ years; body height: $176.8 \pm 2.8 \mathrm{~cm}$; body mass: $67.9 \pm 4.7 \mathrm{~kg}$; $\mathrm{VO}_{2} \max : 62.3 \pm 0.5$ $\mathrm{ml} / \mathrm{kg} / \mathrm{min}$ ), centerbacks ( $\mathrm{n}=4$; age: $19.8 \pm 0.5$ years; body height: $185.5 \pm 7.9 \mathrm{~cm}$; body mass: $79.3 \pm 9.9$ $\mathrm{kg}$; $\mathrm{VO}_{2}$ max: $59.3 \pm 5.6 \mathrm{ml} / \mathrm{kg}^{\prime} \min$ ), midfielders ( $\mathrm{n}=4$; age: $19.8 \pm 0.5$ years; body height: $176.8 \pm 2.8 \mathrm{~cm}$; body mass: $67.9 \pm 4.7 \mathrm{~kg} ; \mathrm{VO}_{2} \max : 69.3 \pm 8.8 \mathrm{ml} / \mathrm{kg} / \mathrm{min}$ ), wingers ( $\mathrm{n}=4$; age: $19.8 \pm 0.5$ years; body height: $169.8 \pm 1.7 \mathrm{~cm}$; body mass: $68.0 \pm 1.2 \mathrm{~kg}$; $\mathrm{VO}_{2} \max : 58.3 \pm 3.4 \mathrm{ml} / \mathrm{kg}^{\prime} \mathrm{min}$ ), forwards $(\mathrm{n}=4$; age: 20 years; body height: $182.3 \pm 2.6 \mathrm{~cm}$; body mass: $74.4 \pm 0.7 \mathrm{~kg}$; $\mathrm{VO}_{2} \max : 55 \pm 11.2 \mathrm{ml} / \mathrm{kg} / \mathrm{min}$ ). The study was applied according to the Helsinki Declaration and objective and possible risks of study were explained to all participiants. Also, it was said that all participiants could leave from study at any time.

\section{Collection of Datas}

The research was performed at pre-season period of young soccer team. The young soccer team had pre-season preparatory camp. The $\mathrm{VO}_{2}$ max and $\mathrm{Vt}$ values of young soccer players were measured by incremental treadmill test. All players were informed about test protocol. Maximum effort was exhibited by players during test protocol. The incremental treadmill test was performed for determining $\mathrm{VO}_{2} \mathrm{max}$ and $\mathrm{Vt}$ values of young soccer players. The initial velocity of incremental velocity test was 10 $\mathrm{km} / \mathrm{h}$. Then velocity was increased by $1 \mathrm{~km} / \mathrm{h}$ at every 3 minutes until exhaustion. The test was finished when players exhausted and didn't continue test due to fatigue. $\mathrm{VO}_{2}$ values during incremental test were measured as breath-by breath by telemetric system (Cosmed $\mathrm{K}_{4} \mathrm{~b}^{2}$, Rome, Italy). Average values of expired gas at every 5 seconds were determined during incremental test. Before incremental test, calibration of oxygen analyzer system was done according to instructions of device calibration. The criterias of $\mathrm{VO}_{2}$ max determination were plateau in $\mathrm{VO}_{2}$ despite constant increase of running velocity and heart rate value passing $90 \%$ of maximal heart rate predicted previously (Taylor et al., 1955). The running velocity of $\mathrm{VO}_{2} \max \left(\mathrm{vVO}_{2} \mathrm{max}\right)$ was determined by identify the lowest running velocity $\mathrm{VO}_{2}$ max occured (Billat and Koralsztein, 1996). Also Vt was determined by incremental treadmill test.

After 3 days from incremental test, players performed treadmill test at $100 \%$ of $\mathrm{vVO}_{2} \max (100 \%$ $\mathrm{vVO}_{2} \max$ test) until exhaustion. Before $100 \% \mathrm{vVO}_{2}$ max test, players performed warm-up for 15 minutes at $60 \%$ of $\mathrm{vVO}_{2}$ max and stretching exercises for 5 minutes. $100 \% \mathrm{vVO}_{2}$ max test was initialized and players were encouraged for maintaining test until exhaustion. $\mathrm{VO}_{2}$ value was measured by gas 
analyzer during test. $\mathrm{VO}_{2}$ plateau was observed at $95 \%$ of $\mathrm{VO}_{2} \max$. Therefore time to achieve $95 \%$ of $\mathrm{VO}_{2} \max \left(\right.$ ta- $95 \% \mathrm{VO}_{2} \max$ ) was time to achieve $\mathrm{VO}_{2} \max \left(\mathrm{ta}-\mathrm{VO}_{2} \max\right.$ ). The ta-95\% $\mathrm{VO}_{2} \max$ and spending time at $\mathrm{VO}_{2} \max \left(\mathrm{t}-\mathrm{VO}_{2} \max \right)$ values of players were computed as below:

$\mathrm{VO}_{2}(\mathrm{t})=\mathrm{VO}_{2 \text { baseline }}+\mathrm{A} \times\left(1-\mathrm{e}^{-(\mathrm{t} / \tau)}\right)($ Mono-exponential function)

At this mono-exponential function, $\mathrm{VO}_{2}(\mathrm{t})$ is oxygen uptake value of time $\mathrm{t}, \mathrm{VO}_{\text {2baseline }}$ is oxygen uptake value measured after warm-up period, $\mathrm{A}$ is amplitude at oxygen uptake value $\left(\mathrm{VO}_{2} \mathrm{max}\right.$ $\mathrm{VO}_{2 \text { baseline) }}$ and $\tau$ is time constant (Barstow and Mole, 1991).

The formula of mono-exponentinal function (equation 1) was regulated as below:

$\mathrm{VO}_{2}(\mathrm{t})=\mathrm{VO}_{2 \text { baseline }}+\mathrm{A} \times\left(1-\mathrm{e}^{-(\mathrm{t} / \tau)}\right)$

For determination of $\mathrm{t}$ (time);

$\mathrm{t}=-\tau \times \ln \left[1-\left(\mathrm{VO}_{2}(\mathrm{t})-\mathrm{VO}_{2 \text { baseline }}\right) / \mathrm{A}\right]$

The ta- $95 \% \mathrm{VO}_{2} \max$ value was equaled to ta- $\mathrm{VO}_{2} \max$ value. Therefore this equation could be expressed as below;

ta- $95 \% \mathrm{VO}_{2} \max =-\tau \times \ln \left[1-\left(95 \% \mathrm{VO}_{2} \max -\mathrm{VO}_{2 \text { baseline }}\right) / \mathrm{A}\right]$

The $\mathrm{t}-\mathrm{VO}_{2} \max$ value was calculated as exhaustion time of test (t-exh.) minus ta- $95 \% \mathrm{VO}_{2} \max$ :

$\mathrm{t}-\mathrm{VO}_{2} \max =\mathrm{t}-\mathrm{exh}-\mathrm{ta}-95 \% \mathrm{VO}_{2} \max$

\section{Analyze of Datas}

The normality distribution of datas was determined by Shapiro-Wilks test and it was seen that datas had normal distribution. The datas of this study were analyzed by SPSS statistical package programme (SPSS 16.0, SPPS Inc., Chicago, USA). One-way analyses of variance (one-way ANOVA) was used for comparing ta-95\% $\mathrm{VO}_{2} \max , \mathrm{t}-\mathrm{VO}_{2} \mathrm{max}$ and Ve values of young soccer players according to playing positions. The differences according to playing positions were determined by Scheffe's Post Hoc tests from one-way analysis of variance (one-way ANOVA). The correlation among ta$95 \% \mathrm{VO}_{2} \mathrm{max}, \mathrm{t}-\mathrm{VO}_{2} \max$ and $\mathrm{Vt}$ values of young soccer players was determined by Pearson correlation coefficient. The level of statistical significiance of all analyzes was assumed at $p<0.05$.

\section{Results}

Table 1. The Values of $\mathrm{VO}_{2} \max , \mathrm{Vt}$, ta- $95 \% \mathrm{VO}_{2} \max , \mathrm{t}-\mathrm{VO}_{2} \max$ and Et Parameters of Young Soccer Players According to Playing Positions. 


\begin{tabular}{|c|c|c|c|c|c|c|c|}
\hline $\begin{array}{l}\text { Playing } \\
\text { Position }\end{array}$ & $\begin{array}{l}\mathrm{VO}_{2} \mathrm{max} \\
(\mathrm{ml} / \mathrm{min})\end{array}$ & $\begin{array}{c}\mathrm{VO}_{2} \mathrm{max} \\
(\mathrm{ml} / \mathrm{kg} / \mathrm{min})\end{array}$ & $\begin{array}{c}\mathrm{Vt} \\
(\mathrm{ml} / \mathrm{min})\end{array}$ & $\begin{array}{c}\mathrm{Vt} \\
(\mathrm{ml} / \mathrm{kg} / \mathrm{min})\end{array}$ & $\begin{array}{c}\mathrm{ta}-95 \% \\
\mathrm{VO}_{2} \max \\
(\mathrm{sec})\end{array}$ & $\begin{array}{c}\mathrm{t}-\mathrm{VO}_{2} \max \\
(\mathrm{sec})\end{array}$ & $\begin{array}{c}\text { Et } \\
\text { (sec) }\end{array}$ \\
\hline Goalkeeper & 4355,4 & 51 & 2818 & 33 & 213 & 171 & 384 \\
\hline Goalkeeper & 4240 & 53 & 2960 & 37 & 223 & 143 & 366 \\
\hline Goalkeeper & 4452 & 53 & 3108 & 37 & 221 & 157 & 378 \\
\hline Mean \pm SD & $4349,1 \pm 106,1$ & $52,3 \pm 1,2$ & $2962 \pm 145$ & $35,7 \pm 2,3$ & $219 \pm 5,3 *$ & $157 \pm 14$ & $376 \pm 9,2$ \\
\hline Fullback & 4158 & 63 & 3102 & 47 & 235 & 122 & 357 \\
\hline Fullback & 4061 & 62 & 2882 & 44 & 243 & 128 & 371 \\
\hline Fullback & 4036,2 & 62 & 3190 & 49 & 283 & 120 & 403 \\
\hline Fullback & 4650 & 62 & 3375 & 45 & 227 & 147 & 374 \\
\hline Mean \pm SD & $4226,3 \pm 287,3$ & $62,3 \pm 0,5$ & $3137 \pm 204,7$ & $46,3 \pm 2,2$ & $247 \pm 24,9$ & $129,3 \pm 12,3$ & $376,3 \pm 19,3$ \\
\hline Center-back & 5540,1 & 59 & 4413 & 47 & 271 & 129 & 400 \\
\hline Center-back & 4114,8 & 54 & 3429 & 45 & 275 & 123 & 398 \\
\hline Center-back & 5025 & 67 & 3225 & 43 & 265 & 124 & 389 \\
\hline Center-back & 4104 & 57 & 3096 & 43 & 261 & 154 & 415 \\
\hline Mean \pm SD & $4696 \pm 709,2$ & $59,3 \pm 5,6$ & $3540,8 \pm 597,4$ & $44,5 \pm 1,9$ & $268 \pm 6,2 *$ & $132,5 \pm 14,6$ & $400,5 \pm 10,8$ \\
\hline Midfielder & 5148 & 78 & 4290 & 65 & 223 & 147 & 370 \\
\hline Midfielder & 6179 & 74 & 4008 & 48 & 257 & 126 & 383 \\
\hline Midfielder & 4431,2 & 58 & 3056 & 40 & 229 & 135 & 364 \\
\hline Midfielder & 4891 & 67 & 3139 & 43 & 275 & 135 & 410 \\
\hline Mean \pm SD & $5162,3 \pm 739,8$ & $69,3 \pm 8,8$ & $3623,3 \pm 618,8$ & $49 \pm 11,2$ & $246 \pm 24,4$ & $135,8 \pm 8,6$ & $381,8 \pm 20,4$ \\
\hline Winger & 4347 & 63 & 3243 & 47 & 223 & 138 & 378 \\
\hline Winger & 3685 & 55 & 3015 & 45 & 271 & 140 & 411 \\
\hline Winger & 3933 & 57 & 2967 & 43 & 243 & 137 & 380 \\
\hline Winger & 3886 & 58 & 3015 & 45 & 257 & 145 & 402 \\
\hline Mean \pm SD & $3962,8 \pm 277,8$ & $58,3 \pm 3,4$ & $3060 \pm 124,1$ & $45 \pm 1,6$ & $248,5 \pm 20,5$ & $140 \pm 3,6$ & $392,8 \pm 16,3$ \\
\hline Forward & 2948 & 40 & 2358 & 32 & 245 & 114 & 359 \\
\hline Forward & 4875 & 65 & 3525 & 47 & 214 & 130 & 344 \\
\hline Forward & 3922 & 53 & 2960 & 40 & 233 & 153 & 386 \\
\hline Forward & 4650 & 62 & 3600 & 48 & 224 & 166 & 390 \\
\hline Mean \pm SD & $4098,8 \pm 868,3$ & $55 \pm 11,2$ & $3110,8 \pm 577,4$ & $41,8 \pm 7,4$ & $229 \pm 13,2$ & $140,8 \pm 23,2$ & $369,8 \pm 22$ \\
\hline Total & $4418,8 \pm 668,7$ & $59,7 \pm 8$ & $3251 \pm 470$ & $44 \pm 6,5$ & $245 \pm 22 * *$ & $138 \pm 15^{* *}$ & $383,1 \pm 18,8$ \\
\hline
\end{tabular}


Table 2. The Values of $\mathrm{VO}_{2} \max , \mathrm{Vt}$, ta- $95 \% \mathrm{VO}_{2} \max , \mathrm{t}-\mathrm{VO}_{2} \max$ and Et Parameters of Young Soccer Players According to Playing Positions During Treadmill Test at $100 \%$ of $\mathrm{vVO}_{2}$ max.

\begin{tabular}{|c|c|c|c|}
\hline $\begin{array}{l}\text { Playing } \\
\text { Position }\end{array}$ & $\begin{array}{l}\mathrm{VO}_{\text {2baseline }} \\
(\mathrm{ml} / \mathrm{min})\end{array}$ & $\begin{array}{c}\mathbf{A} \\
(\mathrm{ml} / \mathrm{min})\end{array}$ & $\begin{array}{c}\mathrm{T} \\
(\mathrm{sec})\end{array}$ \\
\hline Goalkeeper & 650 & 3705,4 & 75 \\
\hline Goalkeeper & 690 & 3550 & 79 \\
\hline Goalkeeper & 515 & 3937 & 77 \\
\hline Mean \pm SD & $618,3 \pm 91,7$ & $3730,8 \pm 194,7$ & $77 \pm 2$ \\
\hline Fullback & 520 & 3638 & 82 \\
\hline Fullback & 630 & 3431 & 86 \\
\hline Fullback & 523 & 3513,2 & 99 \\
\hline Fullback & 515 & 4135 & 79 \\
\hline Mean \pm SD & $547 \pm 55,4$ & $3679,3 \pm 315,5$ & $86,5 \pm 8,8$ \\
\hline Center-back & 750 & 4790,1 & 95 \\
\hline Center-back & 710 & 3404,8 & 98 \\
\hline Center-back & 684 & 4341 & 93 \\
\hline Center-back & 592 & 3512 & 92 \\
\hline Mean \pm SD & $684 \pm 67,1$ & $4012 \pm 666,4$ & $94,5 \pm 2,6$ \\
\hline Midfielder & 484 & 4664 & 77 \\
\hline Midfielder & 620 & 5559 & 89 \\
\hline Midfielder & 678 & 3753,2 & 81 \\
\hline Midfielder & 732 & 4159 & 97 \\
\hline Mean \pm SD & $628,5 \pm 106,6$ & $4533,8 \pm 778,4$ & $86 \pm 8,9$ \\
\hline Winger & 580 & 3767 & 84 \\
\hline Winger & 475 & 3210 & 95 \\
\hline Winger & 489 & 3444 & 85 \\
\hline Winger & 508 & 3378 & 90 \\
\hline Mean \pm SD & $513 \pm 46,7$ & $3449,8 \pm 233,3$ & $88,5 \pm 5,1$ \\
\hline Forward & 570 & 2378 & 88 \\
\hline Forward & 490 & 4385 & 74 \\
\hline Forward & 455 & 3467 & 81 \\
\hline Forward & 521 & 4129 & 78 \\
\hline Mean \pm SD & $509 \pm 48,8$ & $3589,8 \pm 895,7$ & $80,3 \pm 5,9$ \\
\hline Total & $581,8 \pm 92,1$ & $3837 \pm 644,4$ & $85,8 \pm 7,9$ \\
\hline
\end{tabular}


The values $\mathrm{VO}_{2} \max , \mathrm{Vt}$, ta- $95 \% \mathrm{VO}_{2} \max , \mathrm{t}-\mathrm{VO}_{2} \max$ and $\mathrm{t}$-exh parameters at $100 \% \mathrm{v} \mathrm{VO}_{2} \max$ test of young soccer players are presented in Table 1. Also, values of $\mathrm{VO}_{2 \text { baseline, }} \mathrm{A}$ and $\tau$ parameters of young soccer players are presented Table 2. According to correlation analyze results, negative correlation at significiant level between ta-95\% $\mathrm{VO}_{2}$ max and $\mathrm{t}-\mathrm{VO}_{2}$ max was determined $(\mathrm{r}=-0.526$, $\mathrm{p}<0.05)$. There was no significiant correlation between $\mathrm{Vt}$ and other parameters $\left(\mathrm{Ta}-95 \% \mathrm{VO}_{2} \mathrm{max}, \mathrm{t}-\right.$ $\left.\mathrm{VO}_{2} \max \right)(\mathrm{p}<0.05)$.

The results of one-way analysis of variance according to playing positions of young soccer players showed that $\mathrm{t}-\mathrm{VO}_{2}$ max and $\mathrm{Vt}$ parameters didn't differentiate among playing positions ( $\mathrm{p}>0.05$ ). Only ta-95\% $\mathrm{VO}_{2} \max$ parameters differentiated significiantly among playing positions $(\mathrm{F}=3,736$, $\mathrm{p}<0.05)$. In terms of playing positions, it was seen that ta- $95 \% \mathrm{VO}_{2}$ max values of centerbacks were higher than ta-95\% $\mathrm{VO}_{2}$ max values of goalkeepers ( $219 \pm 5,29$ sec., $268 \pm 6,22 \mathrm{sec}$., $\mathrm{p}<0.05$, respectively). There was no significiant difference among other playing positions without difference between goalkeepers and centerbacks ( $p>0.05)$.

\section{Discussion and Conclusion}

It was said that oxygen was derived at onset of constant load exercise by anaerobic energy systems. Oxygen debt occured until $\mathrm{VO}_{2}$ reached plateu level. The ta- $95 \% \mathrm{VO}_{2}$ max parameter meant that $\mathrm{VO}_{2} \max$ was occured. The $\mathrm{t}-\mathrm{VO}_{2}$ max parameter related to balance between ta- $\mathrm{VO}_{2} \mathrm{max}$ and $\mathrm{t}$-exh parameters (Billat et al., 2000). The ta- $95 \% \mathrm{VO}_{2} \max$ parameter had negative correlation with $\mathrm{t}-\mathrm{VO}_{2} \mathrm{max}$ parameter. This meant that higher ta- $95 \% \mathrm{VO}_{2}$ max values caused lower $\mathrm{t}-\mathrm{VO}_{2}$ max values. In terms of $\mathrm{VO}_{2}$ max values, it was seen that $\mathrm{VO}_{2}$ max values of young soccer players $(59,7 \pm 8 \mathrm{ml} / \mathrm{kg} / \mathrm{min})$ were similar to $\mathrm{VO}_{2} \max$ values of middle $(59,8 \pm 1,2 \mathrm{ml} / \mathrm{kg} / \mathrm{min})$ and long $(60,2 \pm 1,5 \mathrm{ml} / \mathrm{kg} / \mathrm{min})$ distance runners found at study of Kilding et al. (2006). This similarity indicates that young soccer and runners may have similar $\mathrm{VO}_{2}$ max and aerobic capacity values. Soccer is a sport needing high aerobic endurance. Therefore, aerobic capacities of young soccer players must be at high level and similarity to aerobic capacity values of middle and long distance runners can be accepted normally.

Dupont et al. (2010) determined oxygen uptake kinetics by mono-exponentinal model and $\mathrm{VO}_{2}$ values measured after severe intensity exercise were lower than $\mathrm{VO}_{2}$ max values of young soccer players measured at our study $(3648,8 \pm 563,7 \mathrm{ml} / \mathrm{kg} / \mathrm{min}, 4418,8 \pm 668,7 \mathrm{ml} / \mathrm{kg} / \mathrm{min}$, respectively). Amateur young soccer players were involved at both studies and young soccer players placing at our study had higher $\mathrm{VO}_{2}$ values than young soccer players of other study. Also, Dupont et al. (2005) determined $\mathrm{VO}_{2} \mathrm{max}$ values of soccer players playing at regional league and these values $(59,4 \pm 4,2 \mathrm{ml} / \mathrm{kg} / \mathrm{min})$ were paralelled to $\mathrm{VO}_{2} \max$ values of our study. Additionally, Dupont et al. (2005) determined relationship between $\mathrm{VO}_{2}$ kinetics and repeated sprints at this study.

Hill et al. (2003) determined $\mathrm{VO}_{2}$ kinetics at treadmill and cycle ergometer by three exponentinal model and $\tau$ value of phase 3 at treadmill test $(86 \pm 39 \mathrm{sec})$. Although determination models 
of $\mathrm{VO}_{2}$ kinetics was different, $\tau$ values were determined as similar. It could be said that $\tau$ values of three exponentinal model might be similar to $\tau$ values of mono-exponentinal model. The $\mathrm{VO}_{2}$ values at threshold level determined at study of Carter et al. (2002) were similar to Vt values of our study $(3036 \pm 199 \mathrm{ml} / \mathrm{kg}, 3251 \pm 470 \mathrm{ml} / \mathrm{kg}$, respectively). The $\mathrm{Vt}$ is a important variable for athletes performance. The $\mathrm{Vt}$ is a deflection point of linearity between minute ventilation and $\mathrm{VO}_{2}$ (Gökbel, 2012). After Vt, ventilation increases exccessively due to carbondioxide $\left(\mathrm{CO}_{2}\right)$ occured by elemination of lactate arising as last product of anaerobic metabolism (MacArdle et al., 2010; Gökbel, 2012). This situation is a factor increased respiratory exchange rate (RER). RER was determined by production of carbondioxide $\left(\mathrm{VCO}_{2}\right)$ divided to $\mathrm{VO}_{2}\left(\mathrm{RER}=\mathrm{VCO}_{2} / \mathrm{VO}_{2}\right)$ and this rate surpasses 1.00 value as $\mathrm{VCO}_{2}$ increases (Gökbel, 2012).

The $\mathrm{Vt}$ is a indicator of endurance performance. There was no correlation between $\mathrm{Vt}$ and other parameters (ta-95\% $\left.\mathrm{VO}_{2} \max , \mathrm{t}-\mathrm{VO}_{2} \max \right)$ in our study. The situation could rise from individual aerobic capacities of young soccer players. It is possible individual differences at aerobic capacity values.

According to playing positions of young soccer players, there was no significiant difference among playing positions in terms of $\mathrm{t}-\mathrm{VO}_{2} \max$ and $\mathrm{Vt}$ parameters ( $\left.\mathrm{p}>0.05\right)$. In terms of ta- $-95 \% \mathrm{VO}_{2} \mathrm{max}$ parameter, it was seen that difference between goalkeeper and centerback playing positions was significiant $(\mathrm{p}<0.05)$. According to Scheffe's test results, no significiant difference among other playing positions was determined ( $>00.05$ ). Davis et al. (1992) determined that predicted $\mathrm{VO}_{2}$ max values of midfield players were the highest values among all playing positions and these values were higher than predicted $\mathrm{VO}_{2}$ max values of centerbacks $(\mathrm{p}<0.05)$. Conversely, it was determined that ta- $95 \% \mathrm{VO}_{2}$ max values were similar to other playing positions without centerbacks. It was said that most of young soccer players had similar aerobic capacity values. The high $\mathrm{VO}_{2} \max$ values of players placing at different playing positions could help them during soccer match and these players could eliminate lactate inducing fatigue. Therefore, performances of players could stay at high level without fatigue.

Rampinini et al. (2010) obtained that Yo-Yo intermittent recovery test level 1 and level 2 performances correlated with $\mathrm{VO}_{2}$ max values positively ( $\mathrm{r}=0,74 ; \mathrm{r}=0,47$, respectively) and $\tau$ values negatively $(r=-0,60 ; r=-0,65$, respectively).According to this findings, the shorter $\tau$ values meant higher aerobic performance. In our study, it was determined that the shorter ta- $95 \% \mathrm{VO}_{2}$ max values caused higher values of $\mathrm{t}-\mathrm{VO}_{2} \max$. These findings were similar to findings obtained by Rampinini et al. (2010). Boone et al. (2012) obtained that fullbacks and midfielders had higher $\mathrm{VO}_{2}$ max values $(61,2 \pm 2,7$ $\mathrm{ml} / \mathrm{kg} / \mathrm{min} ; 60,4 \pm 2,8 \mathrm{ml} / \mathrm{kg} / \mathrm{min}$, respectively) than $\mathrm{VO}_{2} \max$ values of strikers $(56,8 \pm 3,1 \mathrm{ml} / \mathrm{kg} / \mathrm{min})$ centerbacks $(55,6 \pm 3,5 \mathrm{ml} / \mathrm{kg} / \mathrm{min})$ and goalkeepers $(52,1 \pm 5 \mathrm{ml} / \mathrm{kg} / \mathrm{min})$. The fullbacks and midfielders performed many efforts for positional roles as winning ball and tackling during match. Therefore, they must have high level physical capacity and aerobic endurance. These results confirmed this thesis. In terms of ta-95\% $\mathrm{VO}_{2}$ max values, findings of our study didn't report any significiant differences among 
playing positions without significiant difference between goalkeepers and centerbacks. In this regard, our study didn’t agree with study of Boone et al (2012).

The findings of this research revealed significiant negative correlation between ta- $95 \% \mathrm{VO}_{2} \max$ and $\mathrm{t}-\mathrm{VO}_{2}$ max of young soccer players. The $\mathrm{VO}_{2}$ kinetics of young soccer players were determined by mono-exponentinal model at test of $100 \% \mathrm{vVO}_{2}$. The $\mathrm{Vt}$ didn't have any significiant correlation with ta-95\% $\mathrm{VO}_{2} \max$ and $\mathrm{t}-\mathrm{VO}_{2} \max$ parameters. Also, no significiant difference was determined among playing positions in terms of $\mathrm{t}-\mathrm{VO}_{2} \max$ and $\mathrm{Vt}$ parameters . Only, it was seen significiant difference between goalkeepers and centerbacks in terms of ta- $95 \% \mathrm{VO}_{2} \max$ parameter. The many studies at literatüre focused aerobic capacities parameters such as $\mathrm{VO}_{2} \max$ and some of them were parallel to our study in terms of results. The $\mathrm{VO}_{2}$ kinetics are valuable for evaluation of aerobic performance. According to negative correlation between ta- $95 \% \mathrm{VO}_{2 \max }$ and $\mathrm{t}-\mathrm{VO}_{2} \max$ parameters, it can be said that $\mathrm{t}-\mathrm{VO}_{2}$ max parameters depend on ta-95\% $\mathrm{VO}_{2}$ max parameters and reaching to steady-state level as soon as possible during exercise and maintaining exercise at this level were important for aerobic performance within the context of $\mathrm{t}-\mathrm{VO}_{2} \max$.

\section{References}

Al-Hazzaa, H., Al-Muzaini, K., Al-Refaee, S., Sulaiman, M., Dafterdar, M., Al-Ghamedi, A., AlKhuraiji, K. (2001). Aerobic and Anaerobic Power Characteristics of Saudi Elite Soccer Players. Journal of Sports Medicine and Physical Fitness.

Árnason, Á., Sigurdsson, S.B., Gudmundsson, A., Holme, I., Engebretsen, L., Bahr, R. (2004). Physical Fitness, Injuries, and Team Performance in Soccer. Medicine and Science in Sports and Exercise, 36: 278-285.

Bangsbo, J., Lindquist, F. (1992). Comparison of Various Exercise Tests with Endurance Performance During Soccer in Professional Players. International Journal of Sports Medicine, 13: 125-132. Bangsbo, J., Nørregaard, L., Thorsoe, F. (1991). Activity Profile of Competition Soccer. Canadian Journal of Sport Sciences, 16: 110-116.

Barstow, T.J., Mole, P.A. (1991). Linear and Nonlinear Characteristics of Oxygen Uptake Kinetics During Heavy Exercise. Journal of Applied Physiology, 71: 2099-2106.

Bassett, D., Howley, E.T. (2000). Limiting Factors for Maximum Oxygen Uptake and Determinants of Endurance Performance. Medicine and Science in Sports and Exercises, 32: 70-84.

Billat, L.V., Koralsztein, J.P. (1996). Significance of the Velocity at $\mathrm{VO}_{2} \mathrm{max}$ and Time to Exhaustion at This Velocity. Sports Medicine, 22: 90-108. 
Billat, V., Morton, R., Blondel, N., Berthoin, S., Bocquet, V., Koralsztein, J., Barstow, T. (2000). Oxygen Kinetics and Modelling of Time to Exhaustion Whilst Running at Various Velocities at Maximal Oxygen Uptake. European Journal of Applied Physiology, 82: 178-187.

Boone, J., Vaeyens, R., Steyaert, A., Bossche, L.V., Bourgois, J. (2012). Physical Fitness of Elite Belgian Soccer Players By Player Position. Journal of Strength and Conditioning Research, 26: 2051-2057.

Burnley, M., Jones, A.M. (2007). Oxygen Uptake Kinetics as A Determinant of Sports Performance. European Journal of Sport Science, 7: 63-79.

Carter, H., Jones, A.M., Barstow, T.J., Burnley, M., Williams, C.A., Doust, J.H. (2000). Oxygen Uptake Kinetics in Treadmill Running and Cycle Ergometry: A Comparison. Journal of Applied Physiolology, 89: 899-907.

Carter, H., Pringle, J.S., Jones, A.M., Doust, J.H. (2002). Oxygen Uptake Kinetics During Treadmill Running Across Exercise Intensity Domains. European Journal of Applied Physiology, 86: 347354.

Casajús, J.A. (2001). Seasonal Variation in Fitness Variables in Professional Soccer Players. Journal of Sports Medicine and Physical Fitness, 41: 463-469.

Chamari, K., Hachana, Y., Kaouech, F., Jeddi, R., Moussa-Chamari, I., Wisløff, U. (2005). Endurance Training and Testing with the Ball in Young Elite Soccer Players. British Journal of Sport Medicine, 39: 24-28.

Davis, J., Brewer, J., Atkin, D. (1992). Pre-Season Physiological Characteristics of English First and Second Division Soccer Players. Jornal of Sport Sciences, 10: 541-547.

Dupont, G., Millet, G.P., Guinhouya, C., Berthoin, S. (2005). Relationship Between Oxygen Uptake Kinetics and Performance in Repeated Running Sprints. European Journal of Applied Physiology, 95: 27-34.

Dupont, G., McCall, A., Prieur, F., Millet, G.P., Berthoin, S. (2010). Faster Oxygen Uptake Kinetics During Recovery Is Related to Better Repeated Sprinting Ability. European Jornal of Applied Physioologyl, 110: 627-634.

Gökbel, H. (2012). Acute and Chronic Adaptations of the Respiratory System to Physical Exercise. Eurasian Journal of Pulmonology, 14: 9-11.

Helgerud, J., Engen, L.C., Wisloff, U., Hoff, J. (2001). Aerobic Endurance Training Improves Soccer Performance. Medicine and Science in Sports and Exercise, 33: 1925-1931.

Hill, D.W., Halcomb, J.N., Stevens, E.C. (2003). Oxygen Uptake Kinetics During Severe Intensity Running and Cycling. European Journal of Applied Physiolology, 89: 612-618.

Jones, A.M., Poole, D.C. (2005). Oxygen Uptake Kinetics in Sport, Exercise and Medicine. London: Routledge. 
Kemi, O., Hoff, J., Engen, L., Helgerud, J., Wisløff. U. (2003). Soccer Specific Testing of Maximal Oxygen Uptake. Journal of Sports Medicine and Physical Fitness, 43: 139.

Kilding, A.E., Winter, E.M., Fysh, M. A. (2006). Comparison of Pulmonary Oxygen Uptake Kinetics in Middle-and Long-Distance Runners. International Journal of Sports Medicine, 27: 419-426.

McArdle, W.D., Katch, F.I., Katch, V.L. (2010). Exercise Physiology: Nutrition, Energy, and Human Performance. Lippincott Williams \& Wilkins.

Rampinini, E., Sassi, A., Azzalin, A., Castagna, C., Menaspà, P., Carlomagno. D., Impellizzeri, F.M. (2010). Physiological Determinants of Yo-Yo Intermittent Recovery Tests in Male Soccer Players. European Journal of Applied Physiology, 108: 401-409.

Rhodes, E., Mosher, R., McKenzie, D., Franks, I., Potts, J., Wenger, H. (1986). Physiological Profiles of the Canadian Olympic Soccer Team. Canadian Journal of Applied Sport Science, 11: 31.

Strudwick, A., Reilly, T., Doran, D. (2002). Anthropometric and Fitness Profiles of Elite Players in Two Football Codes. Journal of Sports Medicine and Physical Fitness, 42: 239.

Taylor, H.L., Buskirk, E., Henschel, A. (1955). Maximal Oxygen Intake as An Objective Measure of Cardio-Respiratory Performance. Journal of Applied Physiology, 8: 73-80.

Wisloeff, U., Helgerud, J., Hoff, J. (1998). Strength and Endurance of Elite Soccer Players. Medicine and Science in Sports and Exercise, 30: 462-467.

Ordu University

Physical Education and Sports School

Turkey.

E-mail:arierdal@hotmail.com 